\title{
Drug-induced burning mouth syndrome: a new clinico-pathological entity?
}

\author{
Giulio Fortuna $\cdot$ Annamaria Pollio
}

Received: 28 August 2012/Accepted: 21 September 2012/Published online: 30 September 2012

(C) The Author(s) 2012. This article is published with open access at Springerlink.com

Sir,

We read with great interest the letter by Coon et al. [1], recently published in this journal and we would like to focus our attention on the following concerns regarding this report:

1. Burning mouth syndrome is a chronic, idiopathic oral mucosal pain/discomfort, in which no clinical lesions or systemic diseases or other causes, especially drugs are identified $[2,3]$. Some authors have used the term "secondary BMS" to indicate a form of oral complaint caused by local/systemic pathological conditions (including drugs), but even this term seems to be inappropriate, as the term "BMS" automatically excludes any known cause of any type of oropharyngeal complaint. Clinically, it is absolutely mandatory to differentiate between any oral burning, which can have many different causes (including drugs) and BMS, which describes a separate idiopathic clinicopathological entity. Therefore, if this patient's oral burning had been caused by carbidopa/levodopa, it would have been more appropriate to make a diagnosis of "carbidopa/levodopa-induced oral burning" and not "BMS or secondary BMS".

2. If authors have suspected an adverse reaction to a specific drug, a reader might wonder why no one of the adverse drug reaction (ADR) algorithms [4, 5] has been used and no specific guideline [6] has been

G. Fortuna $(\bowtie) \cdot$ A. Pollio

Oral Medicine Unit, Department of Odontostomatological and Maxillofacial Sciences, Federico II University of Naples, Via Pansini 5, 80131 Naples, Italy

e-mail: giulio.fortuna@gmail.com followed to conclusively prove a causative-relationship between oral burning sensation and carbidopa/ levodopa.

3. In addition, one of these ADR algorithms would have helped clinicians in better differentiating between an oral burning induced by carbidopa/levodopa and an oral burning simply as a manifestation of Parkinson's disease (PD). Indeed, considering the high association between PD and oral burning [7], PD might have caused this oral discomfort on its own.

4. Last, but not less important, it is necessary to distinguish an oral burning sensation as a clinical manifestation of an anxiety/depression trait or PD, considering either the association between PD and depressive symptoms (45\%) [8] and anxiety (50\%) [9] or between anxiety, depression and idiopathic BMS [10].

Rebus sic stantibus, it seems hard to establish where does this oral burning really come from (carbidopa/levodopa, PD, anxiety/depression, or a combination of these variables?) This case highlights, for all health care professionals, the need of a more rigorous diagnostic protocol before drawing any conclusion on a possible etiology of any oropharyngeal complaint.

\section{Conflict of interest None.}

Open Access This article is distributed under the terms of the Creative Commons Attribution License which permits any use, distribution, and reproduction in any medium, provided the original author(s) and the source are credited. 


\section{References}

1. Coon EA, Laughlin RS (2012) Burning mouth syndrome in Parkinson's disease: dopamine as cure or cause? J Headache Pain 13:255-257

2. Zakrzewska JM (2010) Medical management of trigeminal neuropathic pains. Expert Opin Pharmacother 11:1239-1254

3. Buchanan JA, Zakrzewska JM (2010) Burning mouth syndrome. Clin Evid pii:1301

4. Agbabiaka TB, Savović J, Ernst E (2008) Methods for causality assessment of adverse drug reactions: a systematic review. Drug Saf 31:21-37

5. Farcas A, Bojita M (2009) Adverse drug reactions in clinical practice: a causality assessment of a case of drug-induced pancreatitis. J Gastrointestin Liver Dis 18:353-358
6. Kelly WN (2003) The quality of published adverse drug event reports. Ann Pharmacother 37:1774-1778

7. Clifford TJ, Warsi MJ, Burnett CA, Lamey PJ (1998) Burning mouth in Parkinson's disease sufferers. Gerodontology 15:73-78

8. Lemke MR (2008) Depressive symptoms in Parkinson's disease. Eur J Neurol 15(Suppl 1):21-25

9. Pontone GM, Williams JR, Anderson KE et al (2009) Prevalence of anxiety disorders and anxiety subtypes in patients with Parkinson's disease. Mov Disord 24:1333-1338

10. Schiavone V, Adamo D, Ventrella G et al (2012) Anxiety, depression, and pain in burning mouth syndrome: first chicken or egg? Headache 52:1019-1025 